\title{
Sexual Stress Immunization Protocols
}

\author{
Stephen Mihailides*, Joe Graffam, Allan Wragg, Andyjim Sinclair and Aikaterini Sisko \\ Department of Psychological Sciences, Swinburne University, Australia
}

\begin{abstract}
The increased attention on methamphetamine as a substance of abuse has proceeded without concurrent innovations deploying its utility as a valid pharmaceutical. Its medicinal properties have not been considered as a basis to understand and correct pathological using patterns. There is a dearth of medical and treatment programs that develop stimulant science for purposeful applications, despite well-known medicinal properties of methamphetamine. The neglected areas of practice are addressed by way of a theoretical development of an approach guided by existing literature. Methamphetamine science has a 100-year journal literature that includes the findings of recent experimental trials, where the pharmacy was found to strengthen the metacognition of agency under known dose structure. Proposed treatment principles are developed, premising the urgency to test those with formulations as the basis of further clinical trials. A defined treatment framework is also presented for deploying metacognition during relapse prevention. Treatment targets for sexual trauma recovery are formulated, because of the pharmacy's potential to support the sexual neuropsychology of the parasympathetic nervous system during recovery. Materials are summarised in five new treatment principles that are developed within combination metacognitive-CBT based frameworks. Psychopharmacological treatment concepts are summarised within the fortified cognition hypothesis. Proposed Sexual Stress Immunisation Protocols (SSIPs), guided by the principles of fortified cognition, preface new trauma and relapse prevention concepts, in their basis for clinical testing.
\end{abstract}

Keywords: Methamphetamine; Psychosexual trauma; Psychopharmacological intervention; Metacognitive therapy

\section{INTRODUCTION}

Despite a century of pharmaceutical science outlining the basis for medicinal benefits of stimulant [1-3], the forensic literature [4-7], though often correctly, identifies methamphetamine as a drug of abuse. Methamphetamine is also a potentially beneficial pharmacy. We consider the theoretical basis for applying stimulant science to medicinal frameworks which include metacognitive components. Means to mitigate sexual trauma, and to inform relapse prevention techniques are emphasized. The article is part of the urgent work needed to determine how to adapt clinical trials [13 ] to the psychology and psychiatry treatment sectors. We consider the adjunctive education, policy mechanisms, and law reform in criminal justice which are also needed urgently for redefined treatment systems. Therefore, the focus is upon defining responsible practice, required to disabuse the science of intervention for this topical treatment area. The utility of recent advances [1-3] reminds of the continued call to develop further bases for experimental trials. Experimental research, with randomized double-blind methodology is appropriate, in order to test the impact of new stimulant treatment frameworks, for their role in improving wellness and for mitigating harm. The proposed role of stimulantfortified metacognition for accelerating resolution of psychosexual traumas is a central focus of this article. Five treatment principles are developed with psychopharmacological basics, for resolution of psychosexual traumas. The treatment principles are organized within the overarching framework of Albert Bandura's theory of Triadic Reciprocal Determinism [8]. Albert Bandura's ideas are developed within Wells and Matthews $[9,10]$ metacognitive theory. Materials are summarized within this framework under the fortified-cognition hypothesis. The second and third focus of the guided theoretical development recognizes the urgencies of relapse-prevention. Theoretical revisions emphasize awe and its affective footprint during, post pharmacological support. The role of mounting metacognition post dosing is explored, for developing means in treatment, to correct cognitive distortions that underpin dependency. Predictions for new interventions

Correspondence to: Stephen Mihailides, Department of Psychological Sciences, Swinburne University, Australia, Tel:+61475062848; E-mail: smihailides@swin.edu.au

Received: January 16, 2019; Accepted: April 04, 2019; Published: May 11, 2019

Citation: Mihailides S, Graffam J, Wragg A, Sinclair A, Sisko A (2019) Sexual Stress Immunization Protocols. J steroids Horm Sci. 4:148. doi:10.35248/2475-319X.19.4.148

Copyright: (C) 2019 Mihailides S, et al. This is an open-access article distributed under the terms of the Creative Commons Attribution License, which permits unrestricted use, distribution, and reproduction in any medium, provided the original author and source are credited. 
to reverse existing dependency are presented in theoretical revisions. A series of additional expectations are defined over the course of the theoretical development. After presenting critical comments, the combination of proposed revisions are the basis of Sexual and Stress Immunisation Protocols (SSIPs), derived from preliminary theoretical induction. SSIPs are the framework for developing further hypotheses for future randomized double-blind experimental trials. SSIP development for metacognitive treatments, organise tenets from both the sympathetic and parasympathetic arms of the central nervous system, into updated exposure-libraries for clinical practice. SSIPs are the basis for extending knowledge of neuropsychological determinism that informs cognitive science.

Theoretical development is initiated with presentation of background materials for Pharmacological Treatment Concepts, the umbrella topic, under which three subsidiary areas are outlined. The Biphasic Dose Hypothesis, the Biochemistry of Neurotransmission and Neurotoxicity theory are topics covered, when appraising methamphetamine pharmacology. After a Critical Review of information, the Fortified Cognition Hypothesis is developed. That precedes the induction of five new principles that are the basis for revision of treatment knowledge. Subsequently, information is compressed, along with Relapse Prevention Innovations, and together, they inform the basis for Sexual Stress Immunization Protocols. Together materials form the foundations of means to redress sexual trauma, and as the basis for structured clinical trials.

\section{PHARMACOLOGICAL TREATMENT CONCEPTS}

The knowledge about the biochemistry of stimulants is reviewed, prior to applying that to the development of treatment principles for methamphetamine. Pharmacological theory about stimulants has existed for over a century. Methamphetamine was first synthesised as a psychomotor stimulant in 1893 [11]. Methamphetamine (methyl amphetamine, methamphetamine, $\mathrm{N}$-methyl-1-phenylpropan-2amine) has two enantiomers, with S-methamphetamine (d-methamphetamine), the more biologically active optical isomer. The terminology, "d" (dextrorotation) and "l" (laevorotation) refers to the rotational properties of the two enantiomers (it is a chiral molecule) in polarised light [11-14]. Amphetamines, as a family of related substances in general, have widespread use in a number of clinical conditions including narcolepsy, orthostatic hypotension, nasal congestion, migraine, asthma, hyperactivity and obesity [12-14]. They are corrective in Attention Deficit and Attention Deficit Hyperactivity Disorders (ADD, ADHD). Mechanism of action, outlined in the "vigilance model of affective disorders", proposes that the pharmaceutical support of stimulants [15] stabilises brain auto regulatory vigilance (brain arousal) through the creation of a stimulating environment. The noradrenergic system is well recognised as implicated with phasic arousal in vigilance [15]. There is some evidence to suggest that depression is more responsive to dopaminergic agents, with stimulants as useful chemical treatments for depressions in bipolar conditions $[12,15,16]$. A great deal is known about the organic chemistry of amphetamine derivatives. Manufactured substances co-occur with many naturally occurring proto-alkaloids such as mescaline and ephedrine that exist in the plant kingdom. The alkaloid backbone for amphetamine is as follows: (Figure 1)

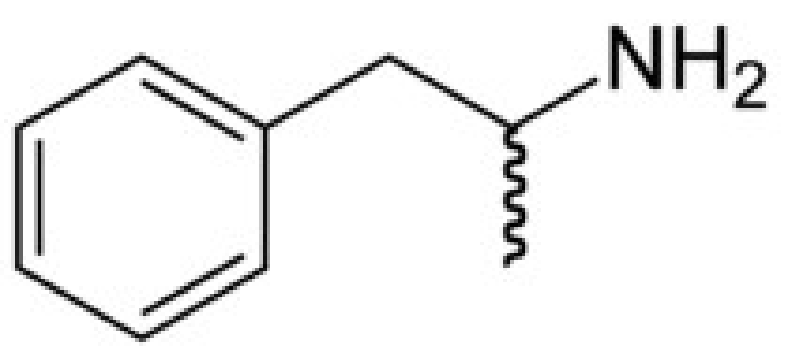

Figure 1: Amphetamine backbone

Chemical modification of the alkaloid at the aromatic ring end (left of picture), or amino group (top right of picture) alters the pharmacological specificity of the compound, considerably [13,14]. Amphetamine is a strong central nervous system stimulant and is also an anorectic agent (i.e. appetite suppressant). The addition of the hydroxy group on the beta carbon (see the peak, to the right of the aromatic ring) reduces both stimulant and anorectic effects. By contrast, addition of a second alpha methyl group, as is the case in methamphetamine, preferentially increases the stimulant properties of an alkaloid (that is, removing a $\mathrm{H}$ bonded to the nitrogen, $\mathrm{N}$, and replacing that with methyl, $-\mathrm{CH} 3$, represented, by convention as a "-" in chemical formulae) Figure 2 below.

Insertion of functional groups on the side chain of amphetamine, or at the terminal amino group, or that target the aromatic ring create anorectic derivatives with reduced stimulant and cardiovascular effects. Aromatic ring substitution by functional groups such as methoxy groups (-O-CH3) have been shown to dramatically alter the pharmacological specificity of the drug, shifting it from a catecholaminergic agent to one that exerts its primary effects upon serotonergic systems [17-19]. It has anti-fatigue effects. At very high dose it induces psychosis. Methamphetamine enhances release, and blocks reuptake of Dopamine (DA), Norepinephrine (NE) and serotonin (5-HT), and is also a monoamine oxidase inhibitor [13-15]. As a result of those effects, methamphetamine acts as an indirect agonist of monoaminergic receptors. At low dose, methamphetamine causes increased motor activity. At high dose, effects include stereotypical movements, and interference with food and water activities that are related to DA, NE and 5-HT systems. In addition, antagonists of dopamine D2 receptors, such as haloperidol and chlorpromazine decrease violence [14,15].

\section{The biphasic dose hypothesis}

Dose and chronicity of use are two important pharmacological parameters, part of the science of pharmacokinetics, that outline a compound's effects upon behaviour, affect and cognition. Therefore, it is important to understand the pharmacological processes that define the dose-related effects of both harmful and non-harmful, medicinal applications of stimulant. The forensic literature provides information about unsupervised, non-optimised, chronic or high-dose of methamphetamine and its impact upon cognition [18-28], with supporting data from Degenhardt et al. [29], who provide prevalence estimates. Dependency, criminality and abuserelated aggression can occur for regular users of high doses. At very high doses, it causes dilated pupils, flushing, restlessness, dry mouth, bruxism, headache, tachycardia, bradycardia, tachypnea, hypertension, hypotension, hyperthermia, diaphoresis, diarrhoea, constipation, blurred vision dizziness, twitching, insomnia, 


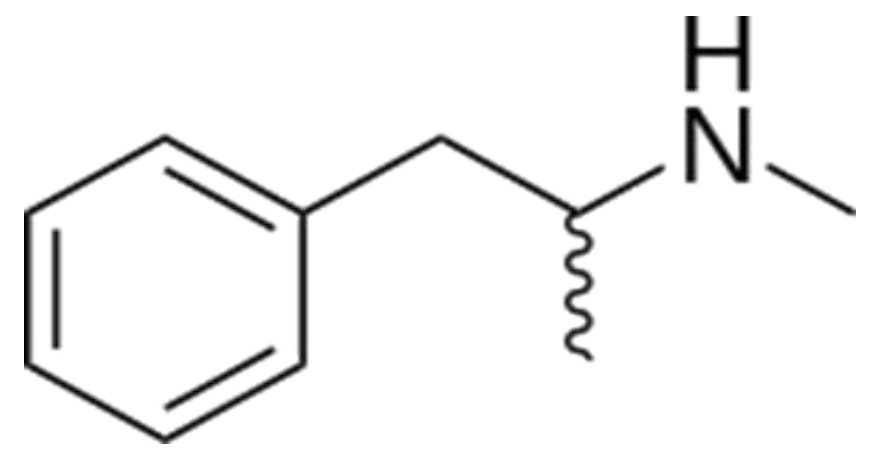

Figure 2: Methamphetamine molecule

numbness, palpitations, arrhythmias, tremors, acne, pallor, seizures, acute myocardial infarction, cerebrovascular accidents and death [12,30]. At high dose, it can induce grandiosity, excessive feelings of power, and invincibility including mania and as noted, psychosis.

However, information about medicinal doses or medicinal uses of methamphetamine [13,31] has existed since the 70s, [31] with clinical trials that first established treatment knowledge that dates back to 1937. Hart et al. [2] theoretical review collated a further sixteen methamphetamine studies (see Table 1., p 590, of the Hart et al. review), which identified stimulant-supported increments to visuospatial perception; reaction time, vigilance, learning/memory; sustained attention, information processing, perceptual speed, psychomotor function, conceptual ability as well as to metacognition [1-3]. Therefore, materials imply that means exist to strengthen understanding of the pharmacokinetic conditions when the stimulant has pharmaceutical capacity.

The relationship between dose and behaviour, studied in experimental research, by Bradley in 1937, explored exogenous methamphetamine dose and impacts on aggression [31]. Data revealed that the stimulant has a biphasic effect, not unitary effects upon aggressive behaviour. That is, their research observed quelling, not exacerbation of aggression under experimental conditions. The early clinical trials noted beneficial treatment effects by administration of amphetamine to aggressive, irritable and hyperactive boys. Results were purportedly repeatedly confirmed by double-blind, placebo-controlled studies. Reductions in aggressive behaviour and improved social interactions were found at doses with 10 to $40 \mathrm{mg} /$ day of d-or l-amphetamine for boys and girls, 5 to 14 years of age, who had been diagnosed with hyperkinetic, autistic, explosive, unsocialised or as emotionally disturbed.

Unfortunately, the forensic literature that outlines the relationship between methamphetamine abuse and aggression [4-7,14,22,32], with its emphasis on pathology and harm, does not trace the causal relationships of biphasic dosing to behaviour. Forensic research is usually correlational, although reliant on neuropharmacological mechanisms developed from animal studies, and from the methodologies such as positron emission tomography to study human brains [11,30]. Forensic research infers causation from the molecular mechanisms, and pharmacokinetics developed in experimental animal studies and from in-vitro methodologies. Coroners' toxicology data, forensic assessment, and post-mortem assessment of dopamine brain markers [30] are also useful to map onto experimental pharmacology research [4-7]. Nonetheless, forensic explanatory models for aggression typically correctly identify misuse of drug, and high-dose, mood-induced changes, psychoses, or withdrawal-related aggression in their explanatory bases [11-
16,24-26]. However, as was noted in Turning Point materials [33], people's violence histories may confound interpretations, and serious dependency was usually observed in patterns of violence. As noted in Turning Point materials for the National drug strategy:

Although there is a perception that all methamphetamine users are violent, this is not the case. Rates of violent crime, although higher than the general population, tend to be restricted to methamphetamine-dependent, multiple drug users with a history of violence. Violence, when it does occur, usually happens when people are paranoid or psychotic. Therefore, hostility and violence is often time-limited, and tends to occur only when symptoms are acute (p.10).

\section{Biochemistry $\mathbb{\&}$ neurotransmission}

Methamphetamine interferes with transport of three biogenic amine neurotransmitters at nerve terminals (dopamine DA, norepinephrine, NE, serotonin, 5-HT, also called monoamines and catecholamines because they contain a catechol group) [30]. Stimulant substitutes for the monoamines in pre-synaptic membrane-bound transporter proteins that ordinarily transport Dopamine (DAT), Noradrenaline (NET), and Serotonin (SERT). The result is release of monoamines from neuronal cytosol via the plasmalemmal transporters, through the process named "reverse transport" [2].

Methamphetamine also interferes with the intracellular transporter, monoamine transporter-2 (VMAT-2), an intracellular vesicle membrane protein, ordinarily associated with establishing reservoirs of monoamines within vesicles. Two mechanisms are thought to act in concert to explain vesicle monoamine depletion [2]. In the first, the weak-base hypothesis posits that, at large amphetamine doses, intracellular concentrations diffuse through vesicle membranes, which disrupt a $\mathrm{pH}$ gradient “...required for vesicular DA sequestration..." [2,p.588]. However, amphetamine is also a VMAT-2 substrate, which competitively inhibits monoamine transport, adding amphetamine to vesicle reservoirs further disrupting the $\mathrm{pH}$ gradient. By the presence of intracellular pools of amphetamine, some of which is distributed into vesicles, DA accumulates in the cytoplasm, and is released into synapses. Synaptic monoamines subsequently stimulate monoamine receptors. In a second mechanism of action, monoamine metabolism is attenuated because methamphetamine inhibits monoamine oxidase [30].

The review by Cruickshank and Dyer [30] reports findings of in-vitro studies, and indicates that methamphetamine is twice as potent at releasing noradrenaline into synapses as dopamine. Its effect was estimated as 60-fold greater on noradrenaline over serotonin release. Overall, amphetamine-like stimulation of the central nervous system causes more potent release of norepinephrine than release of dopamine and serotonin. As was noted in the review in 2012 by Marshall and O'Dell [11], endogenous stimulant affects multiple receptor subtypes for each transmitter system, which have distinct binding affinities.

Other molecular mechanisms [11] that affect neurotransmission include intracellular second-messenger effects, connected to, or influenced by monoamine activity. The central nervous system's complex interacting network of neuronal pathways, with widespread monoamine neuropsychological impacts, means that neurotransmitter changes modulate function of other neurons. Monoamines, for example, are also excitatory to glutamate and inhibitory to Gamma-Amino Butyric Acid (GABA) neurons, 
and baseline dopaminergic activity influences response to amphetamines. Contrary to popular belief, there is also a varied hedonic tone associated with individual-differences in subjective response to ingestion. A low baseline of the D2 dopamine receptor subset is associated with pleasant experience, whereas high baseline density may produce an unpleasant subjective experience.

Methamphetamine's impact on dopamine circuitry and brain reward systems feature prominently in modelling. Neuroanatomical areas affected $[11,30]$ by chemical stimulation include mesolimbic circuitry, and the nigrostriatal pathways. Stimulant-mediated arousal of noradrenergic circuitry has impacts upon pre-frontal cortex, hippocampal function, and memory consolidation. However, the serotonergic circuitry [17], also affected, is widely distributed, and is associated with higher cognitive functions. Serotonin is also involved in the regulation of multiple brain functions such as reward, hyperthermia, respiration, pain perception, sexual behaviour, satiety, impulsiveness, and anxiety.

\section{Neurotoxicity}

Chronic use at high-dose is known to be neurotoxic [11]. The review conducted in 2012 by Marshall and O'Dell [11] is an integration of neurotoxicity research for readers interested in molecular mechanism of methamphetamine-related brain injuries. Their coverage of areas of harm includes evidence for increased production of reactive oxygen and nitrogen species, metabolic compromise, inflammatory responses, alterations in cellular proteins, and blood-brain barrier leakage. Authors' [11] review of experimental animal model methodologies for studying brain injury, included the high-dose 'binge', 'single-day', 'multiple-day' versus 'escalating dose' and 'self-administration' procedures. Binge studies generally provide evidence for monoaminergic markers traced to brain injury, excepting "Self-administration studies in which access to mAMPH...", that is, methamphetamine, "...is limited to 1-2 hours/day showed no lasting changes in monoaminergic markers" [11,p.3]. Hart's et al. [2] review points out that animal neurotoxicity studies apply doses at concentrations that typically exceed those of human using populations. Authors also concluded that, in general, imaging data of research participants fell within the normal, not deficient range, for the studies that were reviewed.

Nonetheless, the neurotoxicity in methamphetamine abuse is a major area of risk associated with intake of high-enough doses. Long-term, high-dose of methamphetamine is neurotoxic to monoamine systems [11,34]. Three lines of evidence support the neurotoxicity hypothesis. In the first, it has been established that levels of dopamine activity affect the enzyme for synthesis of dopamine in a rate-limiting step. Long-term methamphetamine use, decreases enzyme-synthesis. In the second line of evidence, the number of reuptake sites for dopamine is reduced in long-term high-dose usage, and in the third, neuronal degradation has been demonstrated in dopamine rich areas of the brain. For serotonin (5-HT) systems, multiple dosing results in long-lasting reduction to tryptophan hydroxylase activity, an enzyme associated with synthesis of 5-HT, as well as 5-HT content and uptake sites on neurons. However, evidence summarised in the Marshall and O'Dell [11] review suggests effects are reversible for all neurotransmitters at the 1-2year intervals.

\section{Critical comments}

The current relapse prevention $[20,35]$ and harm prevention $[7,21$ -
23] sectors are emphasized in the international response, with criminal industry a serious problem for societies, policy strategy and law enforcement. Unfortunately, a concurrent pressure to diversify treatment frameworks and to fortify the evidence base with updated stimulant science is lacking. Instead, fear about the impacts of drug abuse, and media portrayals of criminality obscure pharmaceutical knowledge. Further, frameworks of law usually do not include plain facility to pressure treatment practitioners and researchers to update their stimulant treatment knowledge, even though professionally accredited courses have responsibility to educate students with new advances in treatment areas. The emphases on a substance of abuse occur despite experimental trials identifying methamphetamine's role in strengthening the metacognition of agency [1-3]. In forensic cohorts, neuropsychological impacts of drug abuse, and brain injuries reported in studies have confounding biases obscuring effects of harm caused only by methamphetamine abuse. Obscuring factors include comorbid traumas predating dependency, socio-economic status, and polysubstance use. There are also expected, serious post-use traumatic exposures such as warrant-based police raids, arrest, prosecution, and exposure to gun violence. Additional psychosocial factors include impacts of recruitment by criminal industries into careercriminal trajectories that cause harm, pressure lives, and shorten life expectancy. Clearly, statistically partitioned impacts of such cohort biases, stratifying their role upon neuropsychological function is needed in additional research. Similarly, pre-withdrawal studies are needed, of those with stable using patterns and lifestyle in order to understand neuropsychology and behavior for that context. Likewise, it is important to understand trauma sequalae, and acute psychosocial stressors, including homelessness, that in combination, contribute to onset of stress-induced brief psychoses. Psychopathologies comorbid in forensic cohorts are distinct from those with psychoses induced by drug abuse, alone. Cruickshank [30], for example, notes that "non-specific environmental stressors such as incarceration, severe insomnia, and heavy alcohol consumption may induce psychotic symptoms during periods of methamphetamine abstinence" (p.1091). Therefore, more research would be useful in order to understand alternative harm-reduction approaches as well that strengthen pharmaceutical benefit, whilst mitigating likelihood of substance abuse. Social policy to assess and redress impacts of criminalization and of belonging to criminal groups is urgently needed. Understanding how the non-criminal social sector more generally affects mental health and dependency onset are equally important. Understanding such harms, that traces to both criminal and non-criminal sectors complicate the measurement of stimulant's footprint upon cognition. Therefore, responding by mitigating harm universally would benefit social wellness, generally. Collective health is a combination of the proportion of difficulties both criminal and non-criminal sectors add to the overall composition of lives. A better appreciation of the new applications for pharmaceutical delivery of methamphetamine is a particular urgency. A number of existing research areas that already exist clarify stimulant properties. Areas include the literature outlining the Hart et al., and Kirkpatrick metacognition experimental studies [1-3] and the review summarizing positive effects of methamphetamine on attention, memory, task performance, and learning [2]. Similarly, the biphasic hypothesis recognizes the potential for constructive stimulant-mediated socio-emotional development. There are terms for pacification of aggression when a stimulant is not abused. However, as a drug of abuse, neurotoxicity data provide the evidence for factors where stimulant misuse contra-indicates a 
pharmaceutical benefit. Therefore, adherence to dosing at optimal levels, over the shorter term and with pauses between short, acutephase usage are means for preventing withdrawal-related aggression and neurotoxicity of drug misuse. Further, future scholarship must assess the contributions to health, when there are means:

i) For accessing psychiatric prescription.

ii) In supervised, remedial clinical contexts.

iii) When access is socially mediated, but responsibly, such that underground use is made obsolete.

Protection afforded by updated knowledge for harm-reduction that occurs in the open cam be distributed more efficiently from cohesive, supportive communities.

\section{THEFORTIFIED-COGNITIONHYPOTHESISAND PSYCHOPHARMACOLOGICAL TREATMENT POTENTIAL OF METHAMPHETAMINE}

Methamphetamine, through its contribution to metacognition [13], means it can be applied to psychological treatments, in combined psychopharmacological interventions. Combining treatment modalities can maximise treatment response, when one modality alone is less efficient. A psychiatric prescription for a pharmacy often includes concurrent referrals for cognitive therapies. Joining pharmaceutical delivery of stimulant with the cognitive therapies likens treatment delivery to those already available for anxiety, depression, mood disorders and psychosis treatment. Purposeful cognitive and pharmaceutical applications can be formulated for methamphetamine, given its pharmacokinetics and impacts upon functioning have been studied for almost a century [31]. As such, we develop the foundation for distinct metacognitive treatment foci, from the combined pharmaceutical and cognitive sciences [1-3,8-10,36-42]. The identified treatment domains are grounded in theoretical frameworks lending themselves to clinical trials. There is nothing really new is the assertion that amphetamines and their derivatives have both known and potentially new treatment potentials for a variety of conditions. Medicinal effects for methamphetamine are predicted to mitigate aggression (at low dose, pursuant to the biphasic hypothesis), for hyper-somniacs, and for a motivational conditions. It is already currently used for attention deficits such as Attention Deficit Hyperactivity Disorder (ADHD), and Attention Deficit Disorder (ADD), under the brand name Desoxyn. It is part of the stimulant family for treating the conditions, with other pharmacies including, Ritalin (methylphenidate), Adderall (amphetamine), Dynavel XR (L-lysinedextroamphetamine). As an anorectic agent, it is also expected to have utility for treating subtypes of obesity, however, because of its pharmacokinetic properties, it is an especially powerful CNS stimulant. Therefore, it can be particularly useful in a subclass of obesity, where a co-morbid medical condition requires it. However, stimulants deployed as adjuncts to cognitive therapies, need to integrate their effects upon motivation, affect, cognition and behaviour, into the structured tasks of the cognitive therapies, delivered in-session. Because methamphetamine supports parasympathetic nervous function, it is expected to be useful for treating sexual pathologies. Sexual function is supported by the parasympathetic, not sympathetic arm (fear circuitry) of the central nervous system. Therefore, it should be particularly useful for treating trauma psychopathology causing hyposexual functioning, where the trauma had onset as a consequence of hate crimes and sexual violence.

The medicinal management requires optimal-range, structured dosing in alliance with cognitive therapies, but for a new area. This treatment area requires experimentally tested knowledge to establish treatment structure. Therefore, clinical trials are required, to establish dose optimisation for the new psychopharmacological principles.

Treatment hypotheses, developed within the metacognitive and cognitive-behavioural traditions, guide the development of five new treatment principles, as premise for structured clinical trials. Thus, the cognitive arm of treatment structure focuses on alliancing behavioural, cognitive and meta-cognitive principles for maximising a person's capacity to divest themselves of hate, trauma and of harm caused by victimisation.

The treatment approach is grounded in methods that foster recovery by empowerment, expected during restoration of a healthy emotional character, with reconstituted, matured sexual competence and confidence as well. The grounding basis for the position derives support from established approaches developed from Albert Bandura's Triadic Reciprocal Determinism [8]. It is a broad framework that combines influences of personal, environmental and behavioural factors (Figure 3 ) into a general treatment-delivery framework. Three levels of analysis are subsumed by cognitive, affective and biological processes in the approach. Albert Bandura's model asserts that intervention may be affected by interceding at any or all points in the system of interacting triadic components. Figure 3 summarises principles in the visual mode.

The fortified-cognition hypothesis, within this framework, predicts that pharmacological support by a stimulant can fortify cognition, and as the basis from which to mount a formalised psychological treatment, in a multidisciplinary-alliance of intervention. The idea is not new. Anti-depressant medications, for example, are deployed in an isomorphic fashion for this purpose. Treatment frameworks using selective serotonin reuptake inhibitors (SSRIs), create fortified psychogenic states, upon which further treatment gains are pursued with a trusted psychologist or psychiatrist. Similarly, for the trauma therapies, Hetrick et al. [43], is a review of treatment for Post-Traumatic Stress Disorder, where stimulant medications were proven to be useful for relieving symptoms of trauma. Likewise, the treatment review [44] of anxiety and mood disorders recognises the advantage of combination cognitive-behavioural-pharmaceutical interventions. Combination treatments return stronger recoveries than patients given medications, alone.

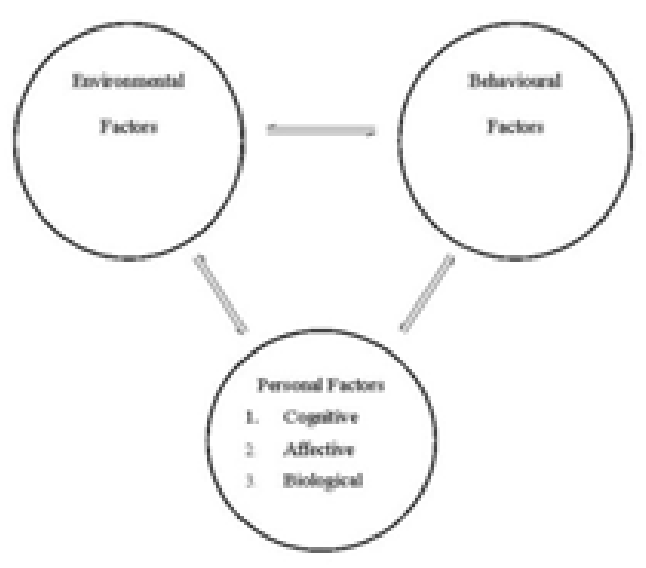

Figure 3: Triadic reciprocal determinism 


\section{Principle 1: Understanding how stimulant fortifies cognition}

Libidinal support: During mild to moderate levels of intoxication, the psychological effects of methamphetamine include alertness, euphoria, increased concentration, energy, self-confidence, sociability, improved self-esteem and supported libido. Methamphetamine transiently repairs mood, self-esteem and confidence. The libidinal enhancement is not particular to methamphetamine. Demonstrated enhanced libidinal drive to erotic stimuli was observed in an experimental study, [45] where researchers administered the stimulant Ritalin (methylphenidate, for treating ADHD) to participants. However, there are individual differences factors expected that are associated with the timing of onset of sexual arousal. That factor set does not have an experimental evidence base. Hypo sexual or hypersexual function can occur in response to stimulant, as is noted on the Ritalin label. Individual differences in response to stimulant, and contextdependent factors that impact the psychopharmaceutical utility of strengthened libido are important to understand. Thus, clinical trials that redress these knowledge deficits areas are important for improving the dearth in the current evidence base.

The libidinal support is extremely important in a trauma treatment framework, where hypo sexuality is part of the avoidance symptom cluster of PTSD. As such, hypo sexuality can potentially reinforce or prolong trauma symptoms because avoidance reinforces pathology. Avoiding engaging in exposure to corrective sexual behaviour will reinforce pathology in situations where the re-experiencing phenomena of abuse include imagery about sexual acts. A sexual or emotional trauma that causes hypo sexual function undermines psychosocial functioning in any case. The combination of emotionally-focussed cognitive work, bolstered by a remedy correcting hyposexual function, is expected to improve general wellbeing. Therefore, relationship satisfaction, and facilitated well-rounded social, vocational and educational goal setting are hypothesised corollaries of corrected sexual function. Similarly, expedient restoration of sexual functioning for couples affected by trauma is expected to prevent premature relationship failure, and to support family functioning. Likewise, correcting libidinal function for single people living with trauma during dating has proposed benefits to self-esteem and to intimacy for friendship development during romantic-relationship development.

Metacognition for developing sexual insight: Experimental studies have demonstrated that intranasal delivery of methamphetamine improves the metacognition of agency in double-blind, controlled experimental conditions [2,3]. Judgments of agency increased, with improvement upon task performance observed for dose ranges as described in the study. Kirkpatrick et al. [3] also recorded dissociation of dose dependency for the positive subjective effects of the substance. Therefore, this pharmaceutical contribution to a person's metacognition should expedite therapy, and assist with psychological treatment formulation, treatment delivery and to strengthen treatment response.

Methamphetamine's augmentation of metacognition means that in principle, it is a valuable resource for that family of psychological therapies that deploy metacognition $[9,10,36-42]$ during treatment delivery. The well-established metacognitive therapies [9] have an associated treatment literature, which evidences efficacy of the metacognitive mode for treating generalised anxiety disorder [41,46], recurrent, persistent depression [39], recurrent major depression with attention training [37], obsessive compulsive disorder [36], social phobia [40], and for treating panic disorder and social phobia [42]. Therefore, the theory for existing metacognitive therapies should provide useful treatment concepts for interventions emphasising stimulant-supported metacognition. The approach also comes with manuals, which are helpful for advancing new treatment targets.

Fortified metacognition that is applied to specific treatment foci, is premised to also result in accelerated rates of insight-development, during treatment delivery. Similarly, increased efficiency is expected for any pleasure and mastery components defined for any associated Cognitive-Behavioural (CBT) intervention foci. High treatment urgencies, such as those central to people suffering with sexual and affective traumas, requires efficient graded-exposure schedules. Strengthened metacognitive facility is expected to assist with exposure tasks designed for urgently mitigating trauma symptoms.

Fortification in other areas: Hypothesised strengthening of treatment efficiency also finds support from the literature that exists which has identified stimulant-enhanced language learning and enhanced memory effects in prior studies $[1-3,47,48]$. Therapy efficiency should benefit from stimulant-supported increments to visuospatial perception; reaction time, vigilance, learning/ memory; sustained attention, information processing, perceptual speed, psychomotor function, conceptual ability as well as to metacognition [1-3].

Principle 2: Optimize exposure by harnessing both sympathetic and parasympathetic arms of the autonomic nervous system

Ordinary cognitive-behavioural exposure therapy for traumas, applied in-session facilitates relief of fear through imaginal-exposure and in-vivo graded-exposure tasks. Treatment delivery includes the formation of the therapeutic alliance, where such exposure schedules are formed under supervision. Brewin [49], is a review of generally accepted neuronal mapping of circuitry involved in the trauma response. He outlines recognised neuropsychological relationships between autobiographical memory, visual memory, the mid-brain structures of the hippocampus and amygdala during the trauma response. Elevations in fear reflect increased activity on the sympathetic arm of the autonomic nervous system [50] implicating the Hypothalamo-Pituitary Axis (HYPAC), with activation of the adrenal glands, raising cortisol and suppressing immunity [51]. Fear extinction during exposure therapy is thought to occur via the hippocampus-dependent form of encoding of autobiographical memory, effective during between-session habituation of CBT. Exposure therapy, effecting therapeutic habituation, also lessons the fear response by attenuation of the automatic, image-based over-activity of amygdala-dependent memory encoding.

Sexual arousal activates the parasympathetic nervous system, with concurrent dampening of the sympathetic nervous system, which means that fear-based circuitry eases during parasympathetic arousal. Therefore, any therapeutic alliancing of the parasympathetic and sympathetic systems, through deployment of behavioural tasks for both arms of the autonomic system, is an obvious way to reclaim wellness more quickly. Deactivating the sympathetic nervous system by adapting pleasure and mastery-based tasks as sexual components to the more traditional graded exposures, is expected to expedite fear-extinction. Trauma, or other causes of hypo-sexual function, implies that redressing sexual function and not just targeting trauma affect will facilitate improvements in romantic and longterm sexual relationships. Repairing sexual capacity should also 
impact one's affective experiences, evoking emotions reflecting sense of confidence, wellbeing and health. Trauma also creates impediments to formation and maintenance of sexual expression in new romantic relationships, and it impacts relationship satisfaction [52-54]. Therefore, a priority exists to define corrective sexual, pleasure and mastery scheduling with the therapeutic CBTmetacognitive focus, in order to restore sexual agency.

\section{Principle 3: Stimulants are known to accelerate resolution of psychoaffective and psychosexual trauma}

The success of another stimulant, ( \pm 3,4-methylenedioxy-Nmethamphetamine, or MDMA, 'ecstasy') has been demonstrated in a clinical trial, for treatment of emotional traumas [55]. Previously, during the early- to mid-part of the $20^{\text {th }}$ century [56] it was a valid adjunct to psychotherapy before partisan lobby groups had it illegalized. The recent clinical trials restore bases for scripted access to this stimulant. MDMA, a compound closely related to methamphetamine, differs only by way of bearing a dioxol functional group (-O-C-O-) on the aromatic end of the amphetamine backbone. It also supports libido, therefore, it is not clear how much impact to treatment gains should be attributed to parasympathetic arousal, for the area not statistically controlled in prior research.

MDMA, in its psychopharmacological action is known as an empathogen and entactogen, that facilitates enhanced intimacy and empathy (it is empathogenic). It also augments pleasurable, tactile physical and sexual contact between people (that is, it is an entactogen). Methamphetamine is less empathogenic, although at optimal dose it sustains empathic function, and early clinical trials found evidence it facilitates social interactions [37]. It is not less entactogenic than MDMA. Methamphetamine enhances confidence and elevates libidinal drive, in an accompanied lowering of inhibitions and anxiety.

The hypothesised trauma-resolution enhancements (Principle 2) are clinical urgencies where ethical treatment response respects expediency of recovery as a fundament of care. Likewise, the expected improvement to libido and to overall confidence ideally match the treatment foci central to psychological therapies targeting sexual traumas. The metacognitive therapies, especially, are expected to be an efficient mode for improving the range of scheduled clinical tasks. Clients can apply such tasks in their lives, by integrating stimulant-augmented sexual psychogenic states into their treatment planning. Pleasure and mastery scheduling, for graded exposure, developed in therapy, can be reviewed and fine-tuned after each deployment of pharmacy-facilitated sexual engagement.

The contribution of traumatic exposure traced to psychopathy and other factors in onset of trauma should be included in the range of materials integrated into clinical trials. That would enable the metacognitive features of treatment to be informed by our recent advances in the understanding of state-psychopathy [57-59], and its known causes of expression in the hate crimes of normal populations. New science that has re-conceptualised our understanding of psychopathy also recognizes means to identify which empathic alliances and attachment bonds are most likely to fortify health. Resolution of trauma, by supported metacognitive focus, that guides strengthened empathic alliances thus should reconstitute lives with more durable overall functioning. We predict that reconstituting empathy with a wider, not narrower range and durability for survivors should be protective against re-traumatisation. This focus places empathy development as a resource in therapy for making sense of why hate crimes occur, and how to quell hate by adaptive response. Survivors enabled in this way create nett benefit to other lives through the guidance to policy, education and child rearing that they often bring to societies.

The CBT-metacognitive alliance, deploying stimulant to quell sympathetic nervous system activity, should result in a re-adaptation to more varied emotional ranges, yielding improved sexual and affective problem solving. Thus, in overall configuration, treatments applying the terms outlined here recognize diversified problem solving by development of insight, language and capacities, formalised by clear focal tasks, impacting sympathetic and parasympathetic nervous system activity and health. Methamphetamine's capacity to open an episode of sexual plasticity is predicted to facilitate sexual wellness, when that process is guided, supported and understood by evidence-based science. The person's acquisition of wisdom through grounding metacognition is hypothesised to facilitate re-engagement with sexual tasks during sexual character reconstitutions.

Of especial relevance, the metacognitive therapies, together with libidinal enhancement of stimulant, should assist with decoding and resolving the impacts of abuse, violence and hatred that so often co-occur with sexual abuse. Sexual traumas often leave the person afflicted with mood disturbance, then residual chronic hatred, rage, and the learned helplessness of the encasing rumination of the re-experienced trauma. In Mihailides, Galligan and Bates [5759] the basis for divesting the afflictions of hate are conclusions developed from review of the impacts of collective psychopathy upon a society's genocide likelihood. A society's overall level of hatred also impels an urgency to divest those hatreds in a variety of settings and roles. Metacognitive therapies are a sound basis to inform treatments to do so. Efficient sexual divestment of hatred is predicted to accelerate maturation of empathic perspective taking, and to prompt client agency, in areas where helplessness delays recovery. Where hatred is causal in trauma onset, sharpening instincts for understanding it, and quelling hatred should be part of treating a trauma. Therefore, resolving trauma by divesting hatred, so that clients do not measure their merit, role or culture by internalised hatred, should preface metacognitive work in therapy. Assistance to strengthen instincts and wisdom about what to do with hate, should equip survivors to clear themselves of self-blame, shame, and to master any of their own hate, directed at others.

\section{Principle 4: Generalizability of treatment goal setting}

Treatment goal setting has nomothetic features, as outlined especially in Principle 2. The nomothetic features are expected to have very wide applicability in ways that traverse systems of role, and merit, and that are relevant cross-culturally. Therefore, such features of methamphetamine-based psychopharmacological intervention are about treatments that focus on sexual development, universally. Mental wellness is strengthened where sexual identity and sexual functioning include acquired sexual resilience in treatment outcome. Sexual capacity is one of two primary assets of mental health, with the second arm being about affective performance. These two primary arms of wellness imply that personal development should also have concurrent idiographic features. That individual focus is required to accommodate the significant variation in personality and presentation of the affective symptoms that accompany a sexual trauma. Therefore, treatment goal setting for sexuo-affective pathology must be responsive, multiply to reflect the way socio-affective and socio-sexual pathologies interact. 
Importantly, treatment formulations for self-esteem, anxietyresolution, and mood-resolution components should reflect attention to metacognitive features of the sexual, not just the affective cognitions that contribute to wellness.

\section{Principle 5: Decoding cognition during methamphetamine affected versus methamphetamine-free states}

The therapist's role is pivotal in how the client's metacognition is harnessed. Trauma is accompanied by cognitive distortions as so noted in the basic diagnostic criteria of PTSD in the Diagnostic and Statistical Manual-V [60]. In an artificially fortified, chemically induced state, cognitive distortions are expected to intrude upon the nature, quality and kind of materials garnered from the person concerned. The therapist's role, by assisting the individual to juxtapose information derived from the medicated versus nonmedicated state, is a guided metacognitive development.

Resolving dose-related cognitive distortions, whilst setting harmmitigating and health-promoting therapeutic sexual goals is recommended, but from short, acute-phase dosing, not continuous dosing. That is, treatment is structured as a pulsing of a series of single optimal doses, followed by cessation of intake and resumption of normal life. Therefore, restoration of autonomy, correcting formerly compromised areas is implied in treatment basics. The autonomy focus of treatment should coincide with matched structured, purposeful therapeutic tasks. A prescribed period between dosing is recommended, with phase-out, or maintenance-prompted medication after the core exposure scheduling is complete. This particular structure is expected to maximise retention of gains of the fortified cognition after cessation of use. Thus, the model of treatment emphasises resolution of dependency and of sexual hatreds in its tenets.

There may be requirement for concurrent access of the person to sleeping medication, because stimulants have the potential to disrupt sleeping patterns, when deployed in acute dosing, before tolerance to the drug occurs. Morning delivery is encouraged to minimise disruption to sleeping patterns, because methamphetamine has a half-life of about twelve hours. Further, deepening appreciation of differential diagnosis, for the potential provision of concurrent mood stabilising medications, is the purview of clinical trials. As is the case with every diagnostic condition in the DSM-V [60], heterogeneity is expected in populations suffering from mental illness, especially where there is dual diagnosis.

\section{Relapse-prevention innovation and guided stimulant use}

McKetin and colleagues [34] state that "...whereas most methamphetamine users take the drug infrequently", then in a noteworthy addition to their comments, authors state: "such recreational patterns of stimulant use have become increasingly apparent in many parts of the world, particularly in the context of dance events". Authors' work [34] notes that socially patterned use is "not typically associated with severe harms", although statistics for recreational-only use were not apparent in their work. They, instead, cite numbers of global users at fifteen to sixteen million. We suspect numbers are understated due to methamphetamine stigma, creating reluctance to disclose, pervasive in contemporary societies. Notwithstanding queries about accuracy of prevalence statistics, it is quite unfortunate that due to the drug's illegal status, such recreational users cannot derive the protective benefits from ritualized, mentored, or responsibly formulated social norms. Such norms do not yet exist. Instead, methamphetamine stigma and courtesy stigmatisation of those who support users are the prevailing social norms. Methamphetamine stigma, not resolved in practitioners, constitutes an iatrogenic factor when stigmatised user groups receive advice or support from such professionals. Users, afraid of prosecution, are less likely to mount protective processes around their social use and they are not enabled to report malpractice of this kind of knowledge deficit to regulatory authorities. Although neurobiological theory [61,62] implicates the limbic system, with onset of dependency, such theories struggle to explain why the majority of users do not develop dependency. Likewise, Marlatt and Gordon [34] in their relapseprevention theory, approach the treatment of dependency after its onset, without emphasising the factors that kept the non-dependent group pathology free. Andrews and Bonta [63], and their pivotal contribution to the forensic psychological literature, prioritise a risk/needs formulation with five rehabilitation principles they apply to extant offending populations. Their work does not assist understanding of the known epidemiology of methamphetamine use, notably, that most people do not develop dependency [34]. The Fortified Cognition hypothesis for stimulant theory, lends itself to innovation for the field of relapse prevention. Its core foundation is grounded in metacognitive functioning, applied during optimized dosing. This no tropic function of the stimulant is hypothesized to rouse affective awe. That is because no tropic or metacognitive insights have vivid impacts during the recognition of insight, and of new ideas. Awe, for the detection of invention, innovation is associated with vitality and vigor of wellness. Pending writing pursues those hypotheses for an overlooked emotion, awe, and its relationship to both metacognition and in the emergence of stable low-end, normal stimulant use.

By contrast, onset of dependency implies a distinct affective footprint for those affected stimulant users. It is suspected that dependencyvulnerability occurs as a problematic response to the sudden onset of clarity durig unguided use. We posit that as metacognition is enhanced mounting awe that dependency vulnerability occurs where a cognitive distortion emerges that becomes reinforced through misadventure. In such contingencies awe becomes fused with the dopamine-related, but pathogenic pleasure effects during use. Such an emphasis places fusion of awe with certain beliefs as cognitive distortions. That is, pleasure and awe are distinct and crucially, pleasure is not happiness. Thus, it is expected that there are individual differences in the cognitive footprint of awe brought by people to stimulant use at its onset. We suspect that it is luck, associated with the quality of rearing, education and cohort exposures, then cultural context, where significant variations in impact of exposure to first use emerge. Therefore, the purpose of clinical trials emphasises the development of a new knowledge base for early detection of risk and for early intervention. Mitigation of that risk is predicted to follow from professional development that formalises terms of reference for corrective, medicinal and for guided, supervised care. The urgency is particularly apparent to redress underground movements of criminal distributorships that can pressure vulnerable cohorts, destructively, and isolate them from services or social alliances that can help prevent harm.

Therefore, the central focus of future writing develops ideas about awe's footprint, and how it appears in adaptive functioning, especially during cessation of use. The temporal appearance of awe in the spectrum of pre- and post-use affect of distinction, premises that human affective instincts with metacognitive grounding, can 
be methodized to quell dependency. It is also anticipated that awe unifies with human spiritual no tropic agency, more generally, and when both supported or unsupported by pharmacies. Insights from that area of metacognition would be expected to guide increments to wellness post and independent of use. Clinical trials should include, therefore, terms for strengthening this area of wellness in participants' lives. In particular, methodising metacognitive intervention so that post-use awe increments the sense of novelty, wellbeing and agency for new life focus, is defined as first priority for theoretical advancement.

Referencing treatment Principle 4 in particular, it is expected that methodizing post-use awe requires the idiographic focus, in order to understand the emotions of unique lives, and how to invest awe into lateral thinking for metacognitive work. Awe is affect that operates adjunctively to any other emotion. For example, awes about grief, versus awes about mastering another's hate for us elicits quite distinct cognition. Awe's utility extends as well into shame resolution, because it guides mastery and fortitude for striving towards gratitude by working from painful originating emotions. Therefore, significant emotional maturation is expected where there is transition from painful affect, to awe in wellness for improved emotional life, by mastery of emotional pain. Awe's adaptability to emotional problem solving is wide. Thus, awe for metacognitive work permits hypotheses, and method for reversing advanced, serious dependency whilst forming new treatment knowledge. Cognitions concurrent with awe are hypothesized to be particularly memorable. It is especially awe for its potential to empower metacognitions that dispatch any helplessness, or poverty of mood that predicates methamphetamine as useful, given how the stimulant supports metacognition.

\section{SEXUAL AND STRESS IMMUNISATION PROTOCOLS}

The fortified cognition hypothesis is the umbrella term developed to organize a theoretical foundation for new treatment concepts. The five new principles of treatment delivery organize distinct areas of theory into subsidiary components, so that hypotheses for treatment trials have clear bases. Together, materials respect the proactive approach taken in Stress Inoculation Training (SIT) [64] where cognitive-behavioural science provides people with toolkits that strengthen their resilience when facing environmental stressors. SIT is thus an approach that targets sympathetic nervous system arousal. Our approach follows on from the traditions of SIT, by adding the metacognitive mode to treatment, and by adding a dimension of care through the parasympathetic arm. The five principles of care identified in the predictions of the fortifiedcognition hypothesis, are summarized for both Sexual and Stress Immunization Protocols (SSIPs). Pending authorship operates from that basis, and formalizes predictions into a methodology that can be applied to randomized double-blind experimental trials. It is anticipated that a database of SSIP tasks will populate a growing library so that they can be made available in treatments on demand.

\section{CONCLUSIONS: IMPLICATIONS FOR POLICY AND LAW REFORM}

One hundred years of pharmacokinetics has demonstrated that methamphetamine as a stimulant, when prescribed correctly, has beneficial impacts on cognition and behaviour. However, methamphetamine-stigma and courtesy-stigmatisation about forwarding clinical reforms have halted the advancement of stimulant science. Thus, the treatment potential for methamphetamine as part of SSIPs has been subvected, preventing access to care. Whilst methamphetamine abuse is a serious matter for a person and their community, that should encourage, not impede the development of evidence-based science for harm mitigation. The view asserted in this review emphasised the quarantining of methamphetamine stigma, so that the important psychopharmacological, and relapseprevention innovations can be developed. We recommend a guided, sensible stimulant regime, with an evidence base for psychopharmacological treatment tasks as prelude to clinical trials. Randomised, double-blind treatment trials to test SSIPs require careful theoretical pre-development, due to the forensic context resulting from stimulant abuse. It is especially important to observe the hate-based dialogue about methamphetamine to understand where that impedes progress in constructive ways. A criminal industry has been created which has impeded methamphetamine science, and undermined deployment of innovations for the field. Further, criminalisation instead creates a resource-intensive and costly, enforcement industry that seeks to tackle a problem downstream. Structuring law and policy systems that assist with resolution of the social problems is required to advance social health. Legalised manufacture of methamphetamine as the pharmaceutical Desoxyn, with greater protection and clear clinical foundations for prescribed medical uses requires systemic law and policy reforms. Education of the populace, in order to shift attitudes and raise awareness about the area is required, as is systemic law reform. New foundations of stimulant science for structured scripted uses, when available assists with social wellness and cohesion. It is also expected that the well-end of the population of infrequent users of stimulant, the recreational group, are an at-risk group, when scientific knowledge is not made available to them. Therefore, assistance more generally is appropriate by having medicalised referral pathways as preventative of harm. Recreational users, who are well, probably access the no tropic of the pharmacy without therapist input. Nonetheless, formalised guidance for stimulant use is encouraged, under supervision, especially for user groups exposed to practices where they are denied harm-prevention advances in stimulant science. Thus, this work's emphasis is to pluralise remedies that methodise extinction and prevention of advanced dependency.

\section{REFERENCES}

1. Hart CL, Gunderson EW, Perez A, Kirkpatrick MG, Thurmond A, Comer SD et al. Acute physiological and behavioral effects of intranasal methamphetamine in humans. Neuropsychopharmacol. 2008;33:18471855.

2. Hart CL, Marvin CB, Silver R, Smith EE. Is cognitive functioning impaired in methamphetamine users? A critical review. J Neuropsychopharmacol. 2012;37:586-608.

3. Kirkpatrick MG, Metcalfe J, Greene MJ, Hart J. Effects of intranasal methamphetamine on metacognition. Psychopharmacol. 2008;197:137144.

4. Darke S, Duflou J, Lappin J, Kaye S. Clinical and autopsy characteristics of fatal methamphetamine toxicity in Australia. J Forensic Sci. 2017;63:1466-1471.

5. Fabian JM. Methamphetamine motivated murder: Forensic psychological/psychiatric \& legal applications in criminal contexts. J Am Psychiatry Law. 2007;35:443-474.

6. Liu Y, Hao B, Shi Y, Xue L, Wang X, Chen Y, et al. Violent offences of methamphetamine users and dilemmas of forensic psychiatric 
assessment. Forensic Sci Res. 2017;2:11-17.

7. Sheridan J, Bennett S, Coggan C, Wheeler A, McMillan K. Injury associated with methamphetamine use: A review of the literature. Harm Red J. 2006;3:14

8. Bandura A. Social foundations of thought and action: A social cognitive theory. Englewood Cliffs, NJ: Prentice-Hall. 1989.

9. Morina N, Normann N. The efficacy of metacognitive therapy: A systematic review and meta-analysis. Front Psychol. 2018;9:1-14.

10. Wells A, Matthews G. Modeling cognition in emotional disorder: the S-REF model. Behav ResTher. 1996;34: 881-888.

11. Marshall JF, O'Dell SJ. Methamphetamine influences on brain and behavior: Unsafe at any speed? Trends in Neurosci. 2012;35:536-545.

12. Asghar K, DeSouza E. Pharmacology and toxicology of amphetamine and related designer drugs. NIDA Res Monogr. 1989;94:1-357.

13. Feldman RS, Meyer JS, Quenzer LF. Principles of neuropsychopharmacology. Sinauer Associates: Massachusetts U.S.A. 1997.

14. Karch SB, Drummer O. Pathology of drug abuse, $2^{\text {nd }}$ edition. Australia: CRC Press. 2012.

15. Hegerl $U$, Hensch $T$. The vigilance regulation model of affective disorders and ADHD. Neurosci Behav Rev. 2014;44:45-57.

16. Carlson PJ, Merlock MC, Suppes T. Adjunctive stimulant use in patients with bipolar disorder: Treatment of residual depression and sedation. Bipolar Disorders. 2004;6:416-420.

17. Lloyd C. Sinning and sinned against: The stigmatisation of problem drug users. UK Drug Policy Commission, London. 2010.

18. Nicosia N, Pacula RL, Kilmer B, Lundberg R, Chiesa J. The economic cost of methamphetamine use in the United States. The meth project foundation and the national institute on drug abuse. RAND Drug Policy Research Center. A Joint Endeavour within RAND Health and Infrastructure, Safety, and Environment. 2005

19. Reinarman C, Levine HG. Crack in America: Demon drugs and social justice. Berkeley, CA, US: University of California Press. 1997.

20. Carroll KM, Rawson RA. Relapse prevention for stimulant dependence. In A Marlatt and DM Donovan (eds.), Relapse Prevention: Maintenance Strategies in the Treatment of Addictive Behaviors, $2^{\text {nd }}$ edition. New York, NY, US: Guilford Press. 2005;130-150.

21. Melnikova N, Welles WL, Wilburn R, Rice WJ, Stanbury M. Hazards of illicit methamphetamine production and efforts at reduction: Data from the hazardous substances emergency events surveillance system. Public Health Rep. 2011;126:116-123.

22. Scott JC, Woods SP, Matt GE, Meyer RA, Heaton RK, Atkinson JH, et al. Neurocognitive effects of methamphetamine: A critical review and meta-analysis. Neuropsychol Rev. 2007;17:275-297.

23. Washton A, Zweben JE. Cocaine and methamphetamine addiction: Treatment, recovery and relapse prevention. New York, NY: Norton. 2009.

24. Bell S, Lucke, JC, Hall WD. Lessons for enhancement from the history of cocaine and amphetamine use. Am J Bioeth: Neurosci. 2012;3:24-29.

25. DeSandre PL. Methamphetamine emergencies. J Gay Lesbian Psychother. 2006; 10:57-65.

26. Fanflik PL, Johnson NE, Troutman DR, Skinner FC. Drug prosecution and prevention across the nation: Prosecutors' perceptions of drug-related crime and strategies to combat the problem in their communities. National District Attorneys Association, USA. 2006.

27. Siebel TM, Mange SA. The Montana meth project: 'Unselling' a dangerous drug. Stanford Law \& Policy Review. 2009;20:405-416.
28. Seiden LS, Kleven MS. Drugs: Toxicity and resulting behavioral changes in response to pharmacological probes. In $\mathrm{K}$ Asghar and $\mathrm{E}$ DeSouza (eds), Pharmacology and Toxicology of Amphetamine and Related Designer Drugs. National Institute on Drug Abuse Research monograph. 1989;94.

29. Degenhardt L, Bucello C, Calabria B, Nelson P, Roberts A, Hall $\mathrm{W}$, et al. What data are available on the extent of illicit drug use and dependence globally? Results of four systematic reviews. Drug Alcohol Depend. 2011;117:85-101.

30. Cruickshank CC, Dyer KR. A review of the clinical pharmacology of methamphetamine. Addiction. 2009;104: 1085-1099.

31. Miczek KA, Tidey JW. Amphetamines: Aggressive and social behavior, 1989. National Institute on Drug Abuse Research Monograph Series 1994;68.

32. Karch SB. Drug abuse handbook, $2^{\text {nd }}$ edition Boca Raton, FL, US CRC Press. 2007.

33. Jenner L, Lee N. Treatment approaches for users of methamphetamine: A practical guide for frontline workers. Canberra: Australian Government Department of Health and Ageing. 2008

34. McKetin R, Hickey K, Devlin K, Lawrence K. The risk of psychotic symptoms associated with recreational methamphetamine use. Drug and Alcohol Rev. 2010;29:358-363.

35. Marlatt GA, Gordon JR. Relapse prevention: Maintenance strategies in the treatment of addictive behaviors. New York: Guilford Press. 1985.

36. Fisher PL, Wells A. Metacognitive therapy for obsessive-compulsive disorder: A case series. J Behav Ther Exper Psych. 2008;39:117-132.

37. Papageorgiou C, Wells A. Treatment of recurrent major depression with attention training. Cogn Behav Pract. 2000;7:407-413.

38. Nisbet J, Shucksmith J. The seventh sense. Scot Educ Rev. 1984; $16: 75-87$.

39. Wells A, Fisher P, Myers S, Wheatley J, Patel T, Brewin CR. Metacognitive therapy in recurrent and persistent depression: A multiple-baseline study of a new treatment. Cognit Ther Res. 2009;33:291-300.

40. Wells A, Papageorgiou C. Brief cognitive therapy for social phobia: A case series. Behav Res Ther. 2001;39: 713-720.

41. Wells A, Welford M, King P, Papageorgiou C, Wisely J, Mendel E. A pilot randomized trial of metacognitive therapy ws applied relaxation in the treatment of adults with generalized anxiety disorder. Behav Res Ther. 2010;48:429-434.

42. Wells A, White J, Carter K. Attention training: Effects on anxiety and beliefs in panic and social phobia. Clin Psychol Psychother. $1997 ; 4: 226-232$.

43. Hetrick SE, Purcell R, Garner B, Parslow R. Combined pharmacotherapy and psychological therapies for Post Traumatic Stress Disorder (PTSD). Cochrane Collaboration. 2012.

44. Otto MW, Smits JA, Reese HE. Combined psychotherapy and pharmacotherapy for mood and anxiety disorders in adults: Review and analysis. Clin Psychol. 2006;12:72-86.

45. Schmid Y, Hysek CM, Preller KH, Bosch OG, Bilderbeck AC, Roger $\mathrm{RD}$, et al. Effects of methylphenidate and MDMA on appraisal of erotic stimuli and intimate relationships. Eur Neuropsychopharmacol. 2015;25:17-25

46. Wells A, King P. Metacognitive therapy for generalized anxiety disorder: An open trial. J Behav Ther Exper Psych. 2006;37:206-212.

47. Breitenstein C, Wailke S, Bushuven S, Kamping S, Zwitserlood P, 
Ringelstein EB, et al. D-amphetamine boosts language learning independent of its cardiovascular and motor arousing effects. Neuropsychopharmacol. 2004;29:1704-1714.

48. Zeeuws I, Soetens E. Verbal memory performance improved via an acute administration of D-amphetamine. Hum Psychopharm. 2007;22:279-287.

49. Brewin CR. A cognitive neuroscience account of posttraumatic stress disorder and its treatment. Behav Res Ther. 2001;39:373-393.

50. McCorry LK. Physiology of the autonomic nervous system. Am J Pharm Educ. 2007;71:78-89.

51. Wilson CKA. Sexual assault and posttraumatic stress disorder: A review of the biological, psychological and sociological factors and treatments. McGill J Med. 2006; 9:111-118.

52. DiMauro J, Renshaw KD. PTSD and relationship satisfaction in female survivors of sexual assault. Psychol Trauma. 2018.

53. Foa EB, Rothbaum BO. Treating the trauma of rape: Cognitivebehavioral therapy for PTSD. New York: Guilford Press. 1998.

54. Foa EB, Rothbaum BO, Riggs DS, Murdock TB. Treatment of posttraumatic stress disorder in rape victims: A comparison between cognitive behavioral procedures and counseling. J Consult Clin Psychol. 1991;59:715-723.

55. Mithoefer MC, Wagner MT, Mithoefer AT, Jerome L, Doblin R. The safety and efficacy of \pm 3 ,4-methylenedioxymethamphetamine- assisted psychotherapy in subjects with chronic, treatment-resistant posttraumatic stress disorder: The first randomized controlled pilot study. J Psychopharmacol. 2011;25:439-452.

56. Reif BS, Oxler F, Freudenmann RW. The origin of mdma ("ecstasy") -separating the facts from the myths. Pharmazie. 2006;61:966-972.

57. Mihailides S, Galligan R, Bates G. Adaptive psychopathy: The quarantine vector and attachment. J Foren Psy. 2017;1-9.

58. Mihailides S, Galligan R, Bates G. Adaptive psychopathy: The quarantine vector and psychopathy induction. J Foren Psy. 2017b;2:1-8.

59. Mihailides S, Galligan R, Bates G. Adaptive psychopathy: Empathy and psychopathy are not mutually exclusive. J Foren Psy. 2017c;2:1-6.

60. American Psychiatric Association. Diagnostic and statistical manual of mental disorders: DSM-5, $5^{\text {th }}$ edition. Arlington, VA: Am J Psych. 2013.

61. Bryant B, Knights K, Salerno E. Pharmacology for health professionals. Mosby/Elsevier Science. Harcourt: Marrickville, N.S.W. 2003.

62. Bullock S, Manias E, Galbraith A. Fundamen pharmacol, $5^{\text {th }}$ edition. Prentice Hall: Australia. 2007.

63. Andrews D, Bonta J. The psychology of criminal conduct. Cincinnati, OH: Anderson Publishing Co. 1994.

64. Meichenbaum D. Stress inoculation training. In P. M. Lehrer, R. L. Woolfolk and W. S. Sime (Eds), Principles and Practice of Stress Management. New York, NY, US: Guilford Press. 2007. 